\title{
Pets' Impact on Your Patients' Health: Leveraging Benefits and Mitigating Risk
}

\author{
Kate Hodgson, DVM, MHSc, CCMEP, Luisa Barton, NP-PHC, DNP, \\ Marcia Darling, Hon. BSc, Viola Antao, MD, CCFP, MHSc, \\ Florence A. Kim, MD, CCFP, and Alan Monavvari, MD, MHSc, CCFP, CHE, CPHQ
}

Over two thirds of Americans live with pets and consider them important members of the family. Pets benefit human health (zooeyia) in 4 ways: as builders of social capital, as agents of harm reduction, as motivators for healthy behavior change, and as potential participants in treatment plans. Conversely, pets can present risks to their owners. They are potential sources of zoonotic disease and injury. Pets can also challenge a family's prioritization of financial and social resources. To activate the benefits of zooeyia and appropriately calibrate and mitigate zoonotic risk, physicians first need to know about the pets in their patients' families. Asking about pets is a simple and feasible approach to assess patients' environmental history and social capital. Asking about pets is a nonthreatening way to build rapport and demonstrates an interest in the whole family, which can improve the physician-patient therapeutic alliance. Physicians can use an interprofessional, collaborative approach with veterinarians to address zoonotic health risks and leverage zooeyia. (J Am Board Fam Med 2015;28:526-534.)

Keywords: Collaboration, Communication, Pets, Primary Health Care, Social Determinants of Health, Zooeyia

Some $68 \%$ of North Americans live with at least 1 pet. ${ }^{1}$ Over three quarters of children in the United States live with pets. Children are more likely to live with a pet than live with their biological father or a sibling. ${ }^{2}$ In Canada, $15 \%$ of families expect to acquire a new pet in the coming year. ${ }^{3}$ Companion animals are a source of health benefits and risks for the families with whom they live. Family physicians who are aware of pets already living with their patients are better able to address this potential.

This article was externally peer reviewed.

Submitted 2 September 2014; revised 13 February 2015; accepted 25 February 2015.

From Continuing Professional Development, Faculty of Medicine (KH), and the Department of Family and Community Medicine (VA, FAK, AM), University of Toronto, Toronto, Ontario, Canada; Nurse Practitioner Prorgam, School of Nursing, Ryerson University, Toronto, Ontario, Canada (LB); and Independent Consultant (MD).

Funding: none.

Conflict of interest: none declared.

Disclaimer: The views expressed in this article are those of the authors and are not an official position of their institutions.

Corresponding author: Kate Hodgson, DVM, MHSc, CCMEP, Continuing Professional Development, Faculty of Medicine, Suite 650, 500 University Ave, Toronto, ON M5G 1V7, Canada (E-mail: kd.hodgson@utoronto.ca).
Pet is a term of endearment reflecting the bond people have with their companion animals. ${ }^{4}$ While dogs and cats are the most popular pets, people keep multiple domestic species as companion animals, including birds, rabbits, hamsters, horses, guinea pigs, ferrets, and gerbils. The dog was the first animal to be domesticated, beginning over 10,000 years ago. ${ }^{5}$ Domestication of animals has been a long and complex process of reciprocal evolutionary pressures affecting both humans and animals. ${ }^{6,7}$ Other animals that have not been domesticated, such as fish, lizards, snakes, and turtles, also are kept as pets. Owners can be strongly bonded with these animals as well.

This article reviews the roles of pets in families, explores zooeyia ${ }^{8}$ (pronounced “zoo-AY-uh")how pets benefit human health-assesses the potential risks of pet ownership, outlines simple communication to identify which patients already have a pet, and describes an interprofessional collaborative approach to improving care for patients with pets.

Evidence of the benefits of pets in families is plentiful, consistent, and reasonably strong (Strength of Recommendation Taxonomy level 
B), ${ }^{9}$ based on multiple, patient-oriented studies. The wide range of protocols, including randomized controlled trials and systematic reviews, represents the full spectrum of Strength of Recommendation Taxonomy levels of quality (1 through 3$).{ }^{9}$ The consistency of results and variation in study quality support a call for rigorous study of human-animal interactions. ${ }^{10}$

The significance of pets to human health has long been acknowledged by many, including the US National Institutes of Health. Their consensus statement on the health benefits of pets concluded with a call for all future studies in human health to consider the presence or absence of a pet in the home and the nature of the relationship with the pet. ${ }^{10}$ Asking about pets opens communication, empowers zooeyia, and can strengthen the physician-patient therapeutic alliance.

\section{Pets in the Family}

People acquire pets for many reasons, including companionship, recreation, and protection. ${ }^{11}$ Most pet owners-regardless of the family's cultureconsider their animal companions part of the family. ${ }^{12}$ Pets are actively integrated with everyday living. ${ }^{4,13,14}$ They participate in family rituals and ceremonies. Many families buy holiday gifts for their pets and often celebrate their birthdays. ${ }^{15}$ In times of family transition, such as illness or death, pets support coping, resilience, and recovery. ${ }^{13-17}$ Military families report that pets provide significant support and stability during the disruption caused by the relocation of a family member. ${ }^{15}$

Contrary to stereotype, pet lovers seldom supplant humans with animals in their relationships; rather, they generalize their capacity for love, empathy, and compassion to all species. ${ }^{17}$ All family members bond with their companion animals, particularly children. The human-animal bond is especially significant for children in single-parent families ${ }^{15,18}$ and those without siblings. ${ }^{19}$ Children often consider relationships with their pets to be more important than some human relationships. ${ }^{20}$ Pets provide comfort and act as confidants. ${ }^{20}$ Pets foster positive psychosocial development in children. ${ }^{17}$ Children with pets demonstrate enhanced empathy, self-esteem, cognitive development, and greater participation in social and athletic activities. ${ }^{17,21}$ They exhibit increased trust, community feeling, sense of safety, and self-confidence. ${ }^{11}$
Psychologists and social workers recognize the importance of pets in families and often include them in their therapeutic approach. ${ }^{17,22}$ The roles of pets evolve through the family's life cycle. Pets can complement the family structure, fitting into the human dynamics and adjusting to the developmental stages of their families. Pets sometimes have the role of a child; sometimes the pet can be a working partner or companion. Pets can also act as replacements for human family members, either augmenting or interfering with human dynamics. ${ }^{14}$

People are deeply affected by the loss of a pet; their responses can echo those to the loss of a human family member or companion. ${ }^{18,22,23}$ Grief may be severe, affecting the owner's health and well-being. For elderly pet owners, the death of a pet can disrupt activities of daily living and distance owners from their social support system. ${ }^{20}$ This normal mourning process does not reflect a pathologic attachment. ${ }^{17,18,23,24}$

\section{Zooeyia: The Health Benefits of Pets}

Zooeyia-the human health benefits of companion animals-affects the physical, emotional, and community spheres. ${ }^{8}$ Zooeyia is taken from the Greek root words for animal (zoion) and health (Hygeia was the ancient Greek goddess of health, the same source as "hygiene"). Zooeyia is the positive inverse of zoonosis (from the same zoion and nosos, or disease). Pets are important companions who affect the health of the families with whom they live. Pets affect determinants of health by enhancing feelings of happiness, security, and self-worth and reducing feelings of loneliness and isolation on a daily basis. ${ }^{25}$ Having pets benefits individual health by encouraging regular exercise and healthy eating patterns, ${ }^{26}$ and by improving physiologic parameters such as hypertension. ${ }^{27}$ Pets augment social interactions in groups, fostering generalized reciprocity and encouraging social trust. ${ }^{28}$ In the community pets catalyze civic engagement, perceptions of neighborhood friendliness, and a sense of community. ${ }^{29}$ Pets can aid a patient's health in 4 ways: as builders of social capital, as agents of harm reduction, as motivators for healthy behavior change, and as active participants in treatment plans.

Pets develop networks among people that lead to cooperation and beneficial outcomes; they build social capital. Walking a dog bridges private and public spaces, facilitating social contact and reci- 
procity between neighbors. ${ }^{30,31}$ Pets encourage participation in organized and casual activities. Pet owners are more civically engaged than people without pets. ${ }^{31}$ Over half of dog owners get to know their neighbors as a result of their pet; over $80 \%$ converse with other pet owners on their walks. $^{31}$

A sense of belonging is an indicator of health. ${ }^{32}$ Pets create feelings of attachment and emotional and social well-being, and decrease feelings of isolation.

Stressful events can escalate in the lives of the elderly as their social supports and physical capabilities diminish. The accumulation of stressful events is associated with increased doctor contacts for patients without pets-but not for pet owners. Pet ownership among seniors enrolled in Medicare reduced their demand for medical services, alleviating costs to the health care system. ${ }^{33}$

Pets can be agents of harm reduction. Patients who engage in high-risk behavior can be adamantly unwilling to harm their pets. Exposure to tobacco smoke is a widely recognized risk factor for many diseases. ${ }^{34}$ Secondhand smoke also affects pets: Cats are susceptible to oral squamous cell carcinoma and mammary cancer; dogs are susceptible to lung cancer and nasal cancer. The risks of pets' exposure to secondhand smoke can motivate pet owners to quit smoking, attempt to quit smoking, encourage other members of the household to quit, and/or prohibit smoking inside the home. ${ }^{35}$ This identifies a novel motivator to amplify smoking cessation messages, of particular significance for smokers who live alone with a pet.

Pets can motivate healthy behavior change. Physical inactivity is such a significant predictor of disease that in one analysis, reducing this risk factor by a mere $10 \%$ would save a projected $\$ 2.1$ billion, or $2.5 \%$ of total direct health care costs. ${ }^{36}$ It is estimated that if adults engaged in 60 minutes of physical activity per day, $33 \%$ of all deaths related to coronary heart disease, $25 \%$ of deaths related to stroke, $20 \%$ of deaths related to type 2 diabetes, and $20 \%$ of deaths related to hypertension would be avoided. ${ }^{37}$ Dog ownership is a reliable indicator of increased physical activity. ${ }^{29}$ Children with dogs spend more time in vigorous physical activity and take more steps per day than those without pets. ${ }^{38}$ Dog owners participate in about $300 \mathrm{~min} / \mathrm{wk}$ of moderate activity, compared with the average 170 $\mathrm{min} / \mathrm{wk}$ of non-dog owners. ${ }^{39}$
Exercise advocates frequently recommend a "buddy system" for physical activity programs. Where a human exercise buddy can both encourage and discourage regular exercise, a dog is a consistently positive influence, initiating exercise and adding enjoyment. ${ }^{39}$ Walking one's dog is also a source of parental pride, a display of the care the owner takes of the animal and of their positive relationship. Parental pride is a motivator to exercise and to interact with others. ${ }^{39}$ Dogs provide a social support system for exercise.

Walking is an accessible exercise for most people, including the obese. More than one third of adults and almost $17 \%$ of youth were obese in 2009 to $2010 .{ }^{40}$ Annual associated medical care costs are estimated at $\$ 147$ billion (2008 US dollars). ${ }^{41}$ Obesity increases the risk of developing serious health conditions such as type 2 diabetes, hypertension, hyperlipidemia, cardiovascular disease, and certain forms of cancer. ${ }^{42}$ Companion animals, particularly dogs, encourage regular exercise, especially walking. Walkers with companion dogs are more likely to maintain any weight loss over a year. ${ }^{39}$

Pets can actively participate in a patient's treatment plan. Chronic diseases place a great burden on patients, their families, the health care system, and society. ${ }^{43}$ Many controllable health risk factors can be positively affected by having pets: physical inactivity, obesity, smoking, hypertension, and the social isolation of chronic disease and psychiatric disease.

One in 3 American adults has high blood pressure. ${ }^{44}$ According to the American Heart Association, the presence of a pet has a significant and positive effect on their owner's cardiovascular reactivity to stress. ${ }^{27}$ Pets provide nonjudgmental social support that buffers pathogenic responses to stress. ${ }^{8,11,21,45}$ Simply stroking a pet lowers blood pressure and serum triglyceride and cholesterol concentrations. ${ }^{8,17,19,46-48}$ In a randomized controlled trial, pets were more effective than angiotensin-converting enzyme inhibitors in controlling hypertension in response to a stressful event. ${ }^{45}$ Cat ownership in particular significantly reduces the risk of cardiovascular disease and associated death. ${ }^{49}$ This holds true regardless of the patient's age, sex, ethnicity, systolic blood pressure, cigarette smoking, diabetes mellitus, serum cholesterol concentration, and body mass index.

For patients with mental health conditions, pets offer multiple benefits. One of 4 adults in the 
Zoonotic disease is species-specific. ${ }^{59}$ Different animal species carry different zoonoses. For example, turtles often carry Salmonella. Zoonotic diseases seen in primary health care include ringworm (dermatophytosis), bite injuries, toxocariasis, Salmonella infection, and avian psittacosis. ${ }^{3,59}$ All mammals are susceptible to dermatophytosis; the most common source for people is infected cats. ${ }^{58}$ Treatment of dermatophytosis is straightforward; however, health care practitioners may not link a patient's recurrent dermatophytosis with a pet's infection. They are simply unaware of pets in the household because they have never asked., ${ }^{3,60}$

Assessment of zoonotic risk from pets should be informed by evidence. For example, most health care providers are aware of toxoplasmosis, which can cause severe and fatal disease in fetuses and immunocompromised individuals. Yet many physicians provide simplistic advice to their pregnant patients, disproportionally focusing on the risk from their cats. ${ }^{61}$ The primary sources of toxoplasmosis are contaminated soil or food. Susceptible people can take simple precautions of proper food hygiene, avoiding handling or eating undercooked or raw meat, and wearing gloves while gardening or cleaning a cat's litter box.

Childhood asthma is a common pet-related concern among family physicians. Asthma has many diverse environmental triggers. ${ }^{62}$ The role of pets may relate to age at exposure. Contact with dogs and cats has a protective effect on respiratory tract symptoms and infections during early childhood development. ${ }^{63}$ Early exposure to pets strengthens the development of the immune system in infants and toddlers and lowers the likelihood of their developing allergies and asthma. ${ }^{11}$ Children in families with dogs have a lower risk of allergic disease; the timing of allergen exposure seems to play a role. ${ }^{64,65}$ Exposure to dogs or cats in the first year of life may actually reduce subsequent risk of allergic sensitization to multiple allergens during childhood. ${ }^{65,66}$

Maintaining pets' health reduces the risk of zoonotic disease in pet-owning families. Both animal and human health care professionals alike play a pivotal role. Vaccinating domestic animals against rabies builds an effective buffer around their families. ${ }^{67}$ Proactive deworming programs for dogs and cats can prevent common intestinal parasites in patients at higher risk. Veterinary examinations can identify and treat zoonotic infections, preventing infection and/or reinfection of members of the household. Veterinarians can provide advice and recommend trainers for a pet's behavioral issues. Appropriate strategies can prevent bite injuries from pets, which is particularly important in families with infants, toddlers, and children.

Pet ownership can create other risks to patients' health by draining family resources: financial, social, and emotional. The cost of caring for a pet may challenge owners' finances, causing an unsustainable imbalance of priorities. Emotional concern for a pet may adversely affect decision making. People may avoid medical care for fear that hospitalization will separate them from their pet. ${ }^{48} \mathrm{~Pa}$ tients have been known to leave a hospital or refuse residential long-term care if their pets are not looked after.

When addressing an issue of health or resource allocation that may be related to an animal, physicians sometimes recommend removing the pet from the home. This should be considered only as a last resort. It will assuredly cause serious conflict within the home. Less than a third of patients will comply because their pet is a member of the family. ${ }^{68}$ It can be devastating to children. ${ }^{69}$ Patients may also mislead caregivers, saying they have disposed of the pet without doing so. ${ }^{68}$ Regardless of outcome, the physician-patient therapeutic alliance can be irreparably damaged. Physicians may become frustrated because the patient (predictably) does not follow their advice. Patients may be distressed that their doctor has such little understanding of their family. Trust is breached.

\section{Asking about Your Patients' Pets}

To activate the benefits of zooeyia and recalibrate zoonotic risk, physicians need to know about pets in their patients' families. Asking about pets can significantly strengthen the physician-patient therapeutic alliance. This simple query demonstrates interest in the patient's entire family and home life. Knowing about pets in the family informs a physician's assessment of a patient's social capital, home environment, and nonmedical determinants of health. Talking about pets is a nonthreatening way to build rapport.

Few resources have been designed to assist health care practitioners in understanding the roles of pets in a family. ${ }^{4,14,22}$ Asking about pets (Figure 1) is a simple and valuable communication ap- 
Figure 1. Asking about pets is a practical, nonthreatening way to open a conversation about the patient's home life.

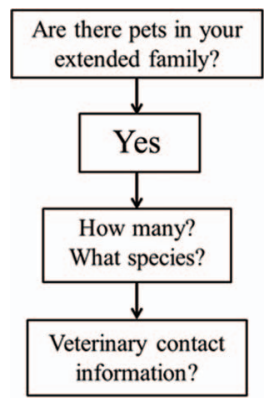

proach. ${ }^{56}$ Enquiries about the patient's home life, including pets, is appropriate when taking a patient's environmental history to update the cumulative patient profile, especially during the initial visit and periodic health reviews. These simple questions are a practical, nonthreatening way to open a conversation about the patient's home life.

- Are there pets in your extended family? If the answer is no, the conversation on this topic is completed. This question is less intrusive than many routinely asked by physicians. The physician's openness on the subject of pets is established. A patient who acquires a pet in the future may recall the interest and advise their physician about the new member of the family.

- How many? What species? Both the benefits and the risks of pets in a patient's life vary by species.

- Veterinary contact information. This creates the potential for interprofessional consultation and collaboration in health matters involving both patients and pets. It will facilitate timely coordinated care, particularly when accompanied by a business card for the patient's veterinarian.

The patient should be advised that no health issue will be discussed with the veterinarian without their express permission to do so, and only if the issue is pertinent to the animal's affect on health care. We understand that permission must be written in specific terms to comply with the Privacy Rule of the US Department of Health and Human Services: "All authorizations must be in plain language, and contain specific information regarding the information to be disclosed or used, the person(s) disclosing and receiving the information, ex- piration, right to revoke in writing, and other data." 70

Issues concerning pets are valid concerns of the family physician. For example, a new kitten may be the source of a child's dermatophytosis. Immunosuppressive therapy elevates the importance of the pet's preventive health care.

\section{An Interprofessional, Collaborative One Health Approach to Recalibrating Risk}

The worldwide One Health ${ }^{71}$ initiative recognizes that the well-being of humans, animals, and the environment are inextricably connected. Collaborative efforts of human and veterinary health care professionals to improve human, animal, and environmental health operate at international, federal, and state levels.

Your pet-owning patients value their relationships with their animals but are unlikely to understand the zoonotic risks of pet ownership, preventive measures, or management strategies. ${ }^{72}$ They may not even know to ask.

Physicians are neither primarily nor solely responsible for managing zoonotic disease. Suspicion or a diagnosis of a zoonotic disease is a manifest opportunity for interprofessional collaboration with the family's veterinarian.

Primary care providers' reluctance to ask about pets may arise from concern that they will not be able to properly advise their patients. Yet patients seek advice from their physician on how to manage their health in the company of their pets. ${ }^{47}$ Many patients do not consider veterinarians to be a source of information on human health, and physicians are generally uncomfortable discussing zoonotic transmission of disease. ${ }^{73}$ They may not recognize and prioritize common, species-specific zoonotic diseases. ${ }^{8}$

The solution lies in interprofessional collaboration. Zoonotic health issues parallel all other situations in which physicians are faced with managing health issues at the edge of their own scope of practice. A veterinarian is a specialist in pet care, just as a pediatrician is in childhood health problems. Family physicians should feel free to confer with and refer to their patients' veterinarians. ${ }^{22}$

Interprofessional collaboration can also leverage zooeyia. A patient may be reluctant to commit to an exercise program without motivation. The primary care provider can suggest the patient consult with 
the veterinarian on how to develop a safe and effective exercise program for the pet that also increases the patient's physical activity. A depressed patient can develop a healthier daily routine by focusing on their pet's exercise requirements and feeding schedule. A hypertensive patient can be encouraged to set aside time every day to spend with their cat.

\section{Conclusion}

Healthy pets contribute to healthy families. Pets enhance emotional well-being and physical health, are sources of social capital, and strongly affect nonmedical determinants of health. Family physicians who know of pets in their patients' families have identified a motivator for the patient to make positive and healthier lifestyle choices and have discovered another potent contributor to treatment plans.

Application in practice is simple, straightforward, and noninvasive. Asking about pets gives physicians a new approach to exploring a patient's home life with a few simple and innocuous questions when taking an environmental history. Further, the demonstrated interest in a pet strengthens the physician-patient therapeutic alliance.

Asking about pets enables realistic recalibration of zoonotic risk. Knowing about pets in the family can influence health care decisions, especially when managing patients with weakened immunity. As in all other circumstances when a primary care provider is at the edge of their scope of practice, interprofessional collaboration can mitigate risk and improve care. Veterinarians are expert in the health care of companion animals and would welcome collaboration with their clients' physicians. ${ }^{74}$ Referrals between these professions, although unusual, ${ }^{74}$ can maximize the quality of patient care. ${ }^{75}$

By knowing about pets in the family, family physicians can activate an existing resource to improve patient care. Just ask.

The authors thank the Women's College Family Practice Peer Support Writing Group for their helpful comments on a draft of this article.

\section{References}

1. 2013/2014 APPA National Pet Owners Survey. Greenwich, CT: American Pet Products Association; 2013. Available from: http://www.americanpetproducts.org/pubs_survey.asp. Accessed May 13, 2015.
2. Reaser JK, Clark EE, Meyers NM. All creatures great and minute: a public policy primer for companion animal zoonoses. Zoonoses Public Health 2008;5 5:385-401.

3. Perrin T. The Business of Urban Animals Survey: the facts and statistics on companion animals in Canada. Can Vet J 2009;50:48-52.

4. Walsh F. Human-animal bonds II: the role of pets in family systems and family therapy. Fam Process 2009;48:481-99.

5. Trut L. Early canid domestication: the farm-fox experiment. Am Sci 1999;87:160-9.

6. Budiansky S. The covenant of the wild: why animals chose domestication. New Haven, CT: Yale University Press; 1999.

7. Ratliff E. Taming the wild. Natl Geogr Mag 2011; 219:35-59.

8. Hodgson K, Darling M. Zooeyia: an essential component of One Health. Can Vet J 2011;52:189-91.

9. Ebell MH, Siwek J, Weiss BD, et al. Strength of Recommendation (SORT): a patient-centered approach to grading evidence in the medical literature. J Am Board Fam Pract 2004;17:59-67.

10. National Institutes of Health. NIH Consensus Development Program. The health benefits of pets. Bethesda, MD: U.S. Department of Health and Human Services; 1987. Available from: http://consensus. nih.gov/1987/1987HealthBenefitsPetsta003html. htm. Accessed November 10, 2014.

11. Smith B. The 'pet effect'-health related aspects of companion animal ownership. Aust Fam Physician 2012;41:439-42.

12. Risley-Curtiss C, Holley LC, Wolf S. The animalhuman bond and ethnic diversity. Soc Work 2006; 51:257-68.

13. Cohen SP. Can pets function as family members? West J Nurs Res 2002;24:621-38.

14. Hodgson K, Darling M. Pets in the family: practical approaches. J Am Anim Hosp Assoc 2011;47:299-305.

15. Beck AM. Review of pets and our mental health: the why, the what, and the how. Anthrozoos 2005;18: 441-3.

16. Allen K. Coping with life changes and transitions: the role of pets. Interactions 1995;13:5-8.

17. Walsh F. Human-animal bonds I: the relational significance of companion animals. Fam Process 2009; 48:462-80.

18. Clements PT, Benasutti KM, Carmone A. Support for bereaved owners of pets. Perspect Psychiatr Care 2003;39:49-54.

19. Wells DL. The effects of animals on human health and well-being. J Soc Issues 2009;65:523-43.

20. McNicholas J, Collis GM. Children's representations of pets in their social networks. Child Care Health Dev 2001;27:279-94.

21. Daly B, Morton LL. An investigation of humananimal interactions and empathy as related to pet 
preference, ownership, attachment, and attitudes in children. Anthrozoos 2006;19:113-27.

22. Hafen M Jr, Rush BR, Reisbig AMJ, McDaniel KZ, White MB. The role of family therapists in veterinary medicine: opportunities for clinical services, education, and research. J Marital Fam Ther 2007; 33:165-76.

23. Toray T. The human-animal bond and loss: providing support for grieving clients. J Ment Health Couns 2004;26:244-59.

24. Sharkin BS, Knox D. Pet loss: issues and implications for the psychologist. Prof Psychol Res Pr 2003; 34:414-21.

25. Sable P. Pets, attachment, and well-being across the life cycle. Soc Work 1995;40:334-41.

26. Vesnaver E, Keller HH. Social influences and eating behavior in later life: a review. J Nutr Gerontol Geriatr 2011;30:2-23.

27. Levine GN, Allen K, Braun LT, et al. Pet ownership and cardiovascular risk: a scientific statement from the American Heart Association. Circulation 2013; 127:2353-63.

28. Putnam RD. Bowling alone: America's declining social capital. J Democr 1995;6:65-78.

29. Brown SG, Rhodes RE. Relationships among dog ownership and leisure-time walking in western Canadian adults. Am J Prev Med 2006;30:131-6.

30. Baum F, Palmer C. 'Opportunity structures': urban landscape, social capital and health promotion in Australia. Health Promot Int 2002;17:351-61.

31. Wood L, Giles-Corti B, Mulsara M. The pet connection: pets as a conduit for social capital? Soc Sci Med 2005;61:1159-73.

32. Statistics Canada. Health Indicators Consensus Conference report: report from the Third Consensus Conference on Health Indicators. Ottawa, Canada: Government of Canada. Available from: http:// www5.statcan.gc.ca/olc-cel/olc.action?objId = 82-230$\mathrm{X} \&$ obj $T$ ype $=2 \&$ lang $=$ en $\&$ limit $=0$. Accessed July 31, 2014.

33. Siegel JM. Stressful events and use of physician services among the elderly: the moderating role of pet ownership. J Pers Soc Psychol 1990;58:1081-6.

34. National Center for Chronic Disease Prevention and Health Promotion. Smoking \& tobacco use: health effects of cigarette smoking. Atlanta: Centers for Disease Control and Prevention. Available from: http://www.cdc.gov/tobacco/data_statistics/fact_sheets/ health_effects/effects_cig_smoking/. Accessed November 9, 2014.

35. Milberger SM, Davis RM, Holm AL. Pet owners' attitudes and behaviors related to smoking and second-hand smoke: a pilot study. Tob Control 2009; 18:156-8.

36. Katzmarzyk PT, Gledhill N, Shephard RJ. The economic burden of physical inactivity in Canada. CMAJ 2000;163:1435-40.

37. Warburton DER, Katzmarzyk PT, Rhodes RE,
Shephard RJ. Evidence-informed physical activity guidelines for Canadian adults. Can J Public Health 2007;98(Suppl):S16-68.

38. Owen CG, Nightingale CM, Rudnicka AR, et al. Family dog ownership and levels of physical activity in childhood: findings from the Child Heart and Health Study in England. Am J Public Health 2010; 100:1669-71.

39. Kushner RF, Blatner DJ, Jewell DE, Rudloff K. The PPET study: people and pets exercising together. Obesity 2006;14:1762-70.

40. Ogden CL, Carroll MD, Kit BK, Flegal KM. Prevalence of obesity in the United States, 2009-2010. NCHS data brief no. 82. January 2012. Bethesda, MD: National Center for Health Statistics. Available from: http://www.cdc.gov/nchs/data/databriefs/db82. pdf. Accessed November 11, 2014.

41. Overweight and obesity: causes and consequences. Atlanta: Centers for Disease Control and Prevention; 2012. Available from: http://www.cdc.gov/obesity/adult/causes/index.html. Accessed November $11,2014$.

42. Crawford AG, Cote C, Couto J, et al. Prevalence of obesity, type II diabetes mellitus, hyperlipidemia, and hypertension in the United States: findings from the GE Centricity Electronic Medical Record database. Popul Health Manag 2010;13:151-61.

43. National Center for Chronic Disease Prevention and Health Promotion. Chronic diseases: the leading causes of death and disability in the United States. Atlanta: Centers for Disease Control and Prevention; 2014. Available from: http://www.cdc.gov/ chronicdisease/overview/. Accessed November 9, 2014.

44. Centers for Disease Control and Prevention (CDC). Vital signs: prevalence, treatment, and control of hypertension-United States, 1999-2002 and 20052008. MMWR Morb Mortal Wkly Rep 2011;60: $103-8$.

45. Allen K, Shykoff BE, Isso JL Jr. Pet ownership, but not ACE inhibitor therapy, blunts home blood pressure responses to mental stress. Hypertension 2001; 38:815-20.

46. Allen KM, Blascovich J, Mendes WB. Cardiovascular reactivity in the presence of pets, friends, and spouses: the truth about cats and dogs. Psychosom Med 2002;64:727-39.

47. Charnetsky CJ, Riggers S, Brennan F. Effect of petting a dog on immune system functioning. Psychol Rep 2004;3:1087-91.

48. McNicholas J, Gilbey A, Rennie A, Ahmedzai S, Dono J, Ormerod E. Pet ownership and human health: a brief review of evidence and issues. BMJ 2005;331:1252-5.

49. Qureshi A, Fareed M, Suri K, Ezzedine MA, Divani AA, Qureshi Z. Cats as domestic pets reduce the risk of cardiovascular diseases: results from the Second $\mathrm{Na}$ tional Health and Nutrition Examination Study Mor- 
tality Follow-Up Study. Abstracts from the 2008 International Stroke Conference. Stroke 2008;39:642-3.

50. CDC report: mental illness surveillance among U.S. adults. Atlanta; Centers for Disease Control and Prevention; 2013. Available from: http://www.cdc.gov/ mentalhealthsurveillance/. Accessed November 11, 2014.

51. Anxiety and Depression Association of America. Facts and statistics. Silver Spring, MD; ADAA. Available from: http://www.adaa.org/about-adaa/ press-room/facts-statistics. Accessed November 11, 2014.

52. Beetz A, Uvnas-Moberg K, Julius H, Kotrschal K. Psychological and psychophysiological effects of human-animal interactions: the possible role of oxytocin. Front Psychol 2012;3:1-15.

53. McCance KL, Huether SE, Brashers VL, Rote NS. Pathophysiology: the biological basis for disease in adults and children. 6th ed. St. Louis: Mosby; 2012.

54. Nagasawa M, Takefumi K, Onaka T, Ohta M. Dog's gaze at its owner increases owner's urinary oxytocin during social interaction. Horm Behav 2009;55:434-41.

55. National Center for Health Statistics. Health, United States, 2013: with special feature on prescription drugs. DHHS publication no. 2014-1232. Hyattsville, MD: U.S. Department of Health and Human Services, Centers for Disease Control and Prevention; 2014. Available from: http://www.cdc.gov/ nchs/data/hus/hus13.pdf. Accessed November 11, 2014.

56. Barton L. Anthrozooeyia: the feasibility and implications of a pet query framework in primary health care practice [dissertation]. Pittsburgh, PA: Carlow University; 2011.

57. Taylor LH, Latham SM, Woolhouse MEJ. Risk factors for human disease emergence. Philos Trans R Soc Lond B Biol Sci 2001;356:983-9.

58. World Health Organization. Meetings on guidelines for drinking-water quality. Geneva: WHO; 2014. Available from: http://www.who.int/water_sanitation_ health/dwq/GDWQ_2013_Repor_microbial_aspects. pdf. Accessed November 11, 2014.

59. Waters J. Healthy pets, healthy people: how to avoid the diseases that pets can spread to people. Paediatr Child Health 2000;5:119-24.

60. Rabinowitz PM, Conti LA. Establishing a new approach to clinical history. In: Rabinowitz, PM, Conti LA, eds. Human-animal medicine: clinical approaches to zoonoses, toxicants, and other shared health risks. Maryland Heights, MO: Saunders Elsevier; 2010:12-7.

61. Rabinowitz PM, Conti LA. Toxoplasmosis. In: Rabinowitz, PM, Conti LA, eds. Human-animal medicine: clinical approaches to zoonoses, toxicants, and other shared health risks. Maryland Heights, MO: Saunders Elsevier; 2010:265-71.
62. Roy A, Downes MJ, Wisnivesky JP. Comprehensive environmental management of asthma and pediatric preventive care. Pediatr Allergy Immunol 2011;22: $277-82$.

63. Bergroth E, Remes S, Pekkanen J, Kauppila T, Buchele G, Keski-Nisula L. Respiratory tract illnesses during the first year of life: effect of dog and cat contacts. Pediatrics 2012;130:211-20.

64. Pohlabeln H, Jacobs S, Bohmann J. Exposure to pets and the risk of allergic symptoms during the first 2 years of life. J Investig Allergol Clin Immunol 2007; 17:302-8.

65. Torrent M, Sunyer J, Garcia R, et al. Early-life allergen exposure and atopy, asthma, and wheeze up to 6 years of age. Am J Respir Crit Care Med 2007; 176:446-53.

66. Ownby DR, Johnson CC, Peterson EL. Exposure to dogs and cats in the first year of life and risk of allergic sensitization at 6 to 7 years of age. JAMA 2002;288:963-72.

67. Centers for Disease Control and Prevention. How can you prevent rabies in people? Atlanta: CDC; 2011. Available from: http://www.cdc.gov/rabies/ prevention/people.html. Accessed November 9, 2014.

68. Ling M, Long AA. Pet dander and difficult-to-control asthma: therapeutic options. Allergy Asthma Proc 2010;31:385-91.

69. Morrow V. My animals and other family: children's perspectives on their relationships with companion animals. Anthrozoos 1998;11:218-26.

70. Summary of the HIPAA privacy rule: authorized uses and disclosures. Washington, DC: U.S. Department of Health and Human Services. Available from: http://www.hhs.gov/ocr/privacy/hipaa/understanding/ summary/index.html. Accessed November 11, 2014.

71. One Health Initiative. One Health Initiative will unite human and veterinary medicine. Available from: http://www.onehealthinitiative.com/index. php. Accessed February 9, 2015.

72. Stull JW, Peregrine AS, Weese JS. Household knowledge, attitudes and practices related to pet contact and associated zoonoses in Ontario, Canada. BMC Public Health 2012;12:1-15.

73. Kahn LH, Kaplan B, Steele JH. Confronting zoonoses through closer collaboration between medicine and veterinary medicine (as 'one medicine'). Vet Ital 2007;43:5-19.

74. Lipton BA, Hopkins SG, Koehler JE, DiGiacomo RF. A survey of veterinarian involvement in zoonotic disease prevention practices. J Am Vet Med Assoc 2008;233:1242-9.

75. Rabinowitz PM, Conti LA. Shared strategies to maximize human and animal health. In: Rabinowitz, PM, Conti LA, eds. Human-animal medicine: clinical approaches to zoonoses, toxicants, and other shared health risks. Maryland Heights, MO: Saunders Elsevier; 2010: 384-93. 